\title{
Influence of Chord Lengths of Splitter Blades on Performance of Small Axial Flow Fan
}

\author{
Guoqi $\mathrm{Li}^{1}$, Lifu Zhu ${ }^{1}$, Yongjun $\mathrm{Hu}^{1}$, Yingzi $\mathrm{Jin}^{*}{ }^{*}$, , Toshiaki Setoguchi ${ }^{2}$ and Heuy Dong Kim ${ }^{3}$ \\ ${ }^{I}$ The Zhejiang Province Key Laboratory of Fluid Transmission Technology, Zhejiang Sci-Tech University, Hangzhou \\ 310018, China \\ ${ }^{2}$ Department of Mechanical Engineering, Saga University, Honjo-machi, Saga, 840-8502, Japan \\ ${ }^{3}$ School of Mechanical Engineering, Andong National University, Seongcheon-Dong 1375, Gyeongdong-RD, Andong, \\ Gyeongsangnuk-DO, 760-749, Korea
}

\begin{abstract}
On the basis of small axial fan with five blades, 6 types of small axial flow fans with different chord lengths splitter blades were designed. Numerical simulation of 6 fan models with splitter blades and prototype fan were done by using Fluent. Based on the obtained simulation results, internal flow characteristics and aerodynamic noise were analyzed and compared. It indicates that: splitter blades with suitable chord length have improved significantly on internal flow characteristics, which inhibits backflow from pressure surface to the suction surface at blade tip and leading edge and restrains flow separation. The 6 model fans are better than prototype fan on aerodynamic noise improvement, but too long or too short chord lengths are both disadvantage to improve aerodynamic noise. The results reveal that $2 / 6,3 / 6$ and $4 / 6$ chord length model have relatively better acoustic characteristics and internal flow characteristics. The research program will offer a reference for structural improvements and noise reduction on small axial flow fan.
\end{abstract}

Keywords: Chord length, Internal flow, Noise, Small axial flow fan, Splitter blade, Static characteristics.

\section{INTRODUCTION}

In daily production and life, fans are widely used in industrial fields, such as aerospace, chemicals, wind power generation and so on. The small axial fan plays an important role in electronic components and other functional elements by cooling and ventilation. If the heat is released insufficiently, the components will do not work properly even are damaged. For example, if computer CPU cooling is not sufficient, the temperature of CPU will be higher and higher, which will directly lead to computer shutting down and affecting the normal operation. Hence, small axial fan plays a vital role on special occasions.

Many studies on small axial fan have been done, Lin and Tsai conduct research on small axial fans and establish an excellent system that is a fusion of aerodynamics, noise and electro-mechanical assessment program, which could be used as an important tool for fan designers [1]. Also, Lin and Tsai establish a design program that integrates cascade theory and inverse design for small axial fan. By design conditions and geometric program, an overall configuration of fan that meets the performance requirements was generated [2].

Many scholars have conducted research on the blow performance, aerodynamic noise and internal flow field of

*Address correspondence to this author at the Faculty of Mechanical Engineering \& Automation, Zhejiang Sci-Tech University, Hangzhou 310018, China; Tel: +86 57186843348; Fax: +86 571 86843348;

E-mail: jin.yz@163.com small axial fan. Li et al. have studied aerodynamic characteristics of small axial fan, which reduces fan noise by perforating on the blade, and the results show that the noise decreases at blade tip clearance and the exit area [3]. Numerical calculation for small axial CPU fan has been performed by Kang et al. via computational fluid dynamic software NUMECA. At the same time, combined with the results of wind tunnel tests AMCA, they obtained internal flow characteristics of fan. By comparing the numerical results with the experimental results, it denotes that they are nearly consistent in a wide range of flow rate [4].

In researching on the fans, the scholars have proposed many optimized solutions to improve blow performance and reduce the fan noise. It is an ideal optimization program that adding the splitter blade in centrifugal turbomachinery, which is relatively rare in the axial turbomachinery. Yuan et al. carry on research about impeller of centrifugal compressor, and design centrifugal impeller with splitter blades. The results of internal flow simulation show that the splitter blade can effectively control the flow separation. What's more, it also reveals that the length of the splitter blade has effects on the impeller efficiency, and a suitable splitter blade length can effectively suppress the secondary flow and improve efficiency [5]. Cai and Luo conduct numerical simulation of centrifugal impeller flow field, analyze two main variables (number of blades and applying Long-short blade structure). Additionally, it denotes that a reasonable number of blades can reduce the secondary flows, and applying Long-short blade structure is effective to improve the fluid flow [6]. Through the experiment and numerical simulation on forward-curved centrifugal fan with splitter 
blades, the result shows that changing the circumferential position of splitter blade has a significant effect on fan performance, and an appropriate length of the splitter blade can increase total pressure coefficient of the fan [7].

This paper draws reference on the splitter blade technology in centrifugal turbomachinery, and applies it to small axial flow fan. Based on different chord lengths of splitter blades, 6 types of small axial fan models with splitter blades are designed, and static characteristics, internal flow characteristics and aerodynamic noise are analyzed.

\section{FAN MODEL AND GEOMETRIC PARAMETERS}

The small axial flow fan prototype is shown in Fig. (1). The outer diameter of fan is $85 \mathrm{~mm}$, blades 5 , rotated speed $3000 \mathrm{rpm}$, hub ratio 0.72 .

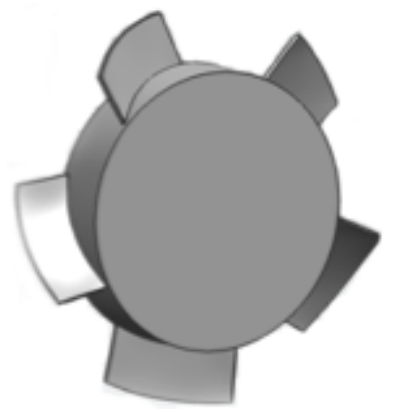

Fig. (1). Prototype fan.

Taken the fan in Fig. (1) as prototype, splitter blades of different chord lengths are designed in this paper. With the help of graphic design methods, 6 types of small axial fan models with different chord lengths splitter blades are built, as shown in Fig. (2). Chord lengths of splitter blades in Fig. (2a) and that of the blades in prototype fan are the same. The whole chord length of blade is divided into 6 equivalent segments, the chord lengths of splitter blades in 5 other fan models are $5 / 6,4 / 6,3 / 6,2 / 6$ and $1 / 6$ chord length, respectively corresponding to Fig. (2b-f). In order to distinguish easily, the fan model in Fig. (2a) is called as $6 / 6$ chord length.

\section{COMPUTATIONAL REGION AND GRID}

As shown in the Fig. (3), the computational domain is divided into 4 regions, namely, rotation area, tip clearance area, inlet extension area and outlet extension area. The center of the fan is set as the origin of coordinates. 8 red noise monitoring points are shown in Fig. (3), whose positions in the $\mathrm{Z}$ direction are shown in Table $\mathbf{1}$.

In Fig. (3), it takes Moving Reference Frame as the reference coordinate of rotation area. Mass flow inlet is applied as inlet boundary condition, and outlet boundary condition is pressure outlet. What's more, in computational domain, it sets the no-slip condition as boundary condition for the solid walls such as fan.

The left picture in Fig. (4) shows the grids of whole computational domain, and structural grid is applied in inlet extension area (yellow) and outlet extension area (blue), whose mesh node spacing is 3 . However, the tip clearance area (pink) and the rotation area of fan are unstructured mesh, and the spacing is set to 1 . Fan rotating area is covered by blade tip clearance area, which is difficult to distinguish from the figure. The right picture in Fig. (4) is a schematic diagram of superficial grid about fan.

\section{NUMERICAL SIMULATION AND RESULT ANALYSIS}

\subsection{Numerical Calculation Method}

Finite volume method is used to conduct flow field calculation of fans, and RNG $k-\varepsilon$ model is chosen as turbulence model in this research. To improve the numerical

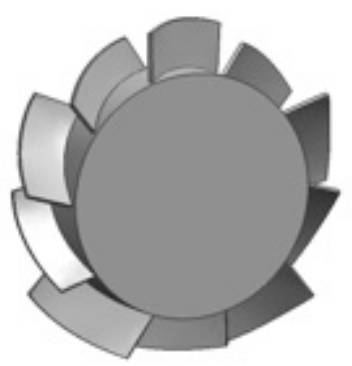

(a) $6 / 6$ chord length

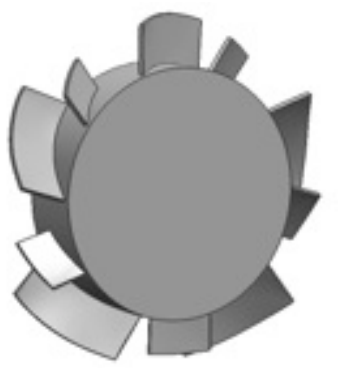

(d) 3/6 chord length

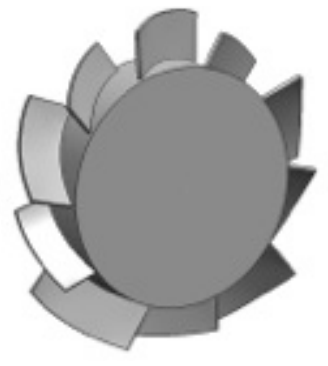

(b) $5 / 6$ chord length

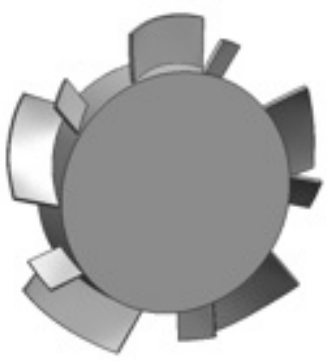

(e) 2/6 chord length

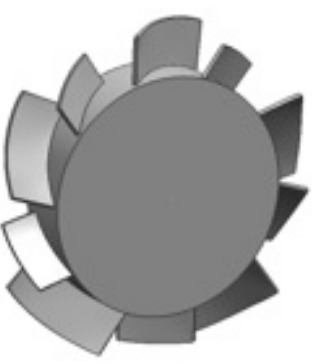

(c) $4 / 6$ chord length

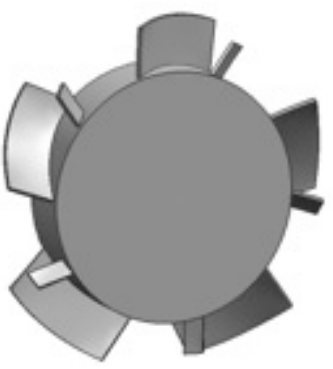

(f) $1 / 6$ chord length

Fig. (2). Fan models with splitter blades of different chord length. 


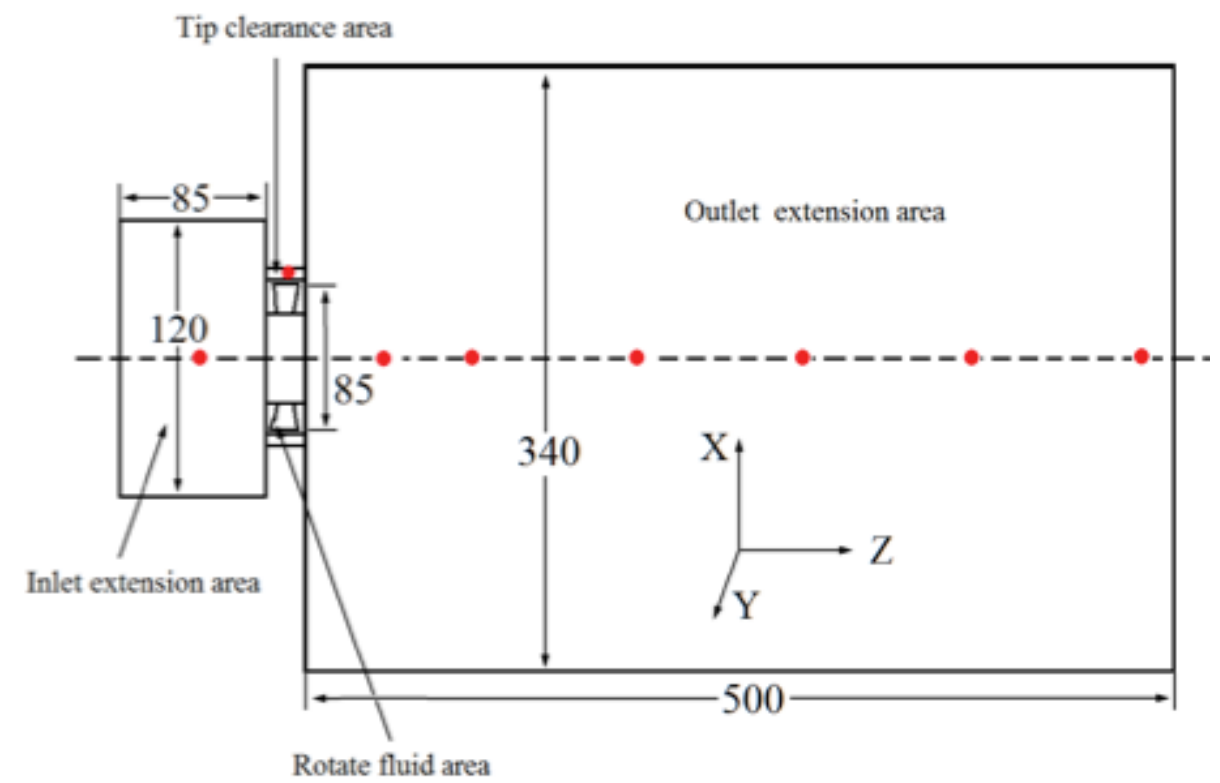

Fig. (3). Computational domain and distribution of noise monitoring points

Table 1. $\quad \mathrm{Z}$ directional distribution of noise monitoring points.

\begin{tabular}{|l|c|c|c|c|c|c|c|c|}
\hline Monitoring Points & 1 & 2 & 3 & 4 & 5 & 6 & 7 & 8 \\
\hline Z Coordinate/mm & -50 & 0 & 50 & 100 & 200 & 300 & 400 & 500 \\
\hline
\end{tabular}

accuracy, second order upwind scheme is applied in governing equations.
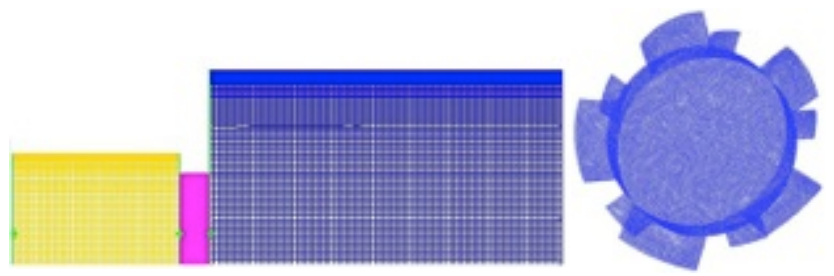

Fig. (4). Grid of computational domain and superficial gird of fan.

The process of Obtaining of aerodynamic noise includes mainly calculation and post-processing. The calculation process contains the following steps: (1) Steady calculation of flow field is done, whose results are as the early field of large eddy simulation (LES); (2) Unsteady calculation is conducted in the early field. In calculation, the pressure and velocity coupling adopts PISO format and the time step of calculation is $2 \times 10^{-5} \mathrm{~s}$; (3) After the pressure field keeps stable, the noise model(FW-H) is introduced to obtain the noise. Regard as processing part, it needs FFT to deal with pressure signal for previous results, and the spectral distribution will be obtained.

\subsection{Static Characteristics}

For the purpose of obtaining the Static pressure-Flux $(\Psi$ $\Phi)$ dimensionless curves and the Efficiency-Flux $(\eta-\Phi)$ dimensionless curves, steady numerical calculations are applied to 6 types of small axial fan models and prototype fan. Dimensionless curves are obtained from following equation:
$\Phi=\frac{4 Q_{m}}{\rho \pi D^{2} u}$

$\psi=\frac{2 \Delta P}{\rho u^{2}}$

In the equation, $Q_{m}$ is mass flow rate, $D$ the outer diameter of the fan, $u$ the circumferential speed of the fan outer periphery, $\Phi$ mass flow coefficient, and $\Psi$ static pressure coefficient.

The Fig. (5) is Static pressure-Flux $(\Psi-\Phi)$ dimensionless curves for 6 types of small axial fan models.

From Fig. (5), it indicates that: (1) static pressure coefficient about 7 types of fans overall decrease with mass flux coefficient increasing; (2) When mass flux coefficient $\Phi$ is smaller than $0.043\left(Q_{m}=0.004 \mathrm{~kg} / \mathrm{s}\right)$, the static pressure situation of prototype fan overall is slightly better than the other models. But when $\Phi$ is larger than 0.043 , the static coefficient of 6 models with splitter blades are higher than that of prototype fan. Besides, the trend is more obvious with flux coefficient increasing; (3) when it is a high flux coefficient, static coefficient of $6 / 6$ chord length and $5 / 6$ chord length is smaller than that of four other model fans. Comprehensively, static coefficient of 4/6 chord length, 3/6 chord length and 2/6 chord length are better than that of other models.

Fig. (6) is the Efficiency-Flux $(\eta-\Phi)$ dimensionless curves for 6 types of small axial fan models and prototype fan. As can be seen from Fig. (6), the most efficient point about the 7 kinds of fans all occurs in the operating point that flux coefficient $\Phi$ was $0.06465\left(Q_{m}=0.006 \mathrm{~kg} / \mathrm{s}\right)$. About the point, the flux coefficient will be operating point in detail below. 


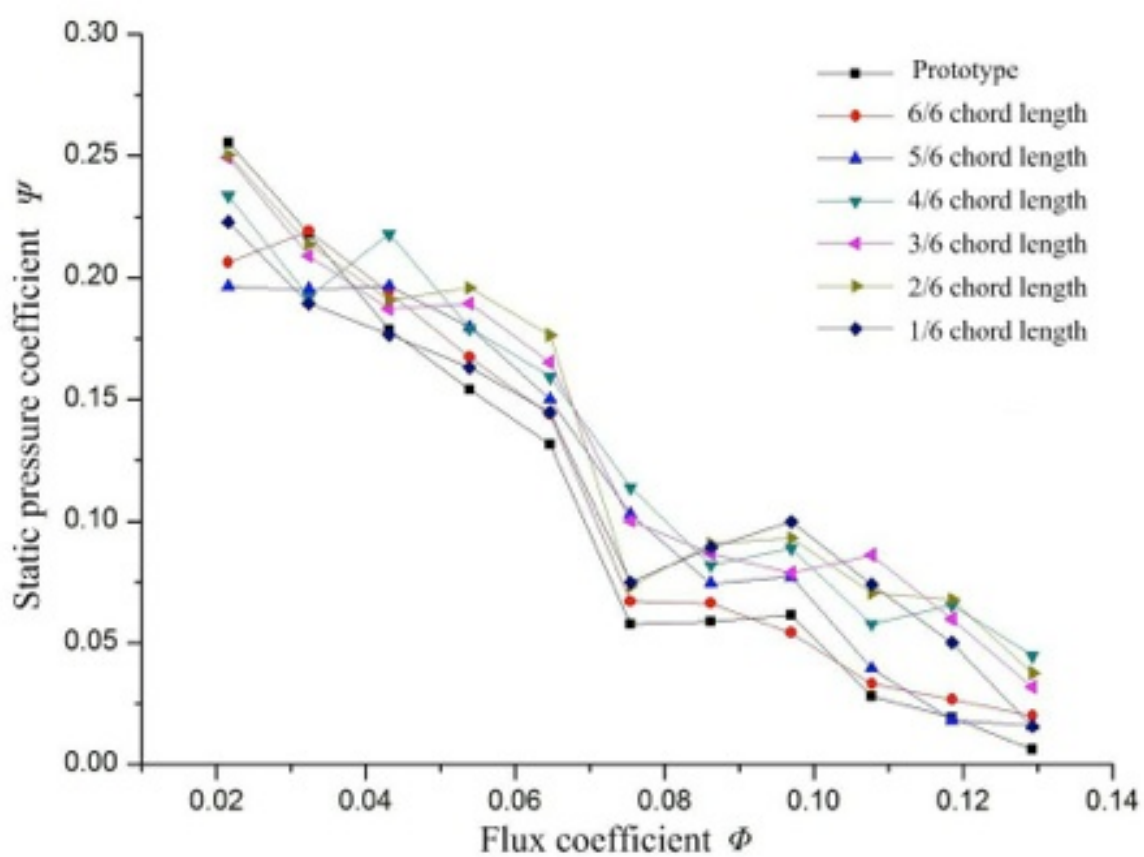

Fig. (5). Static Pressure-Flux $(\Psi-\Phi)$ dimensionless curves.

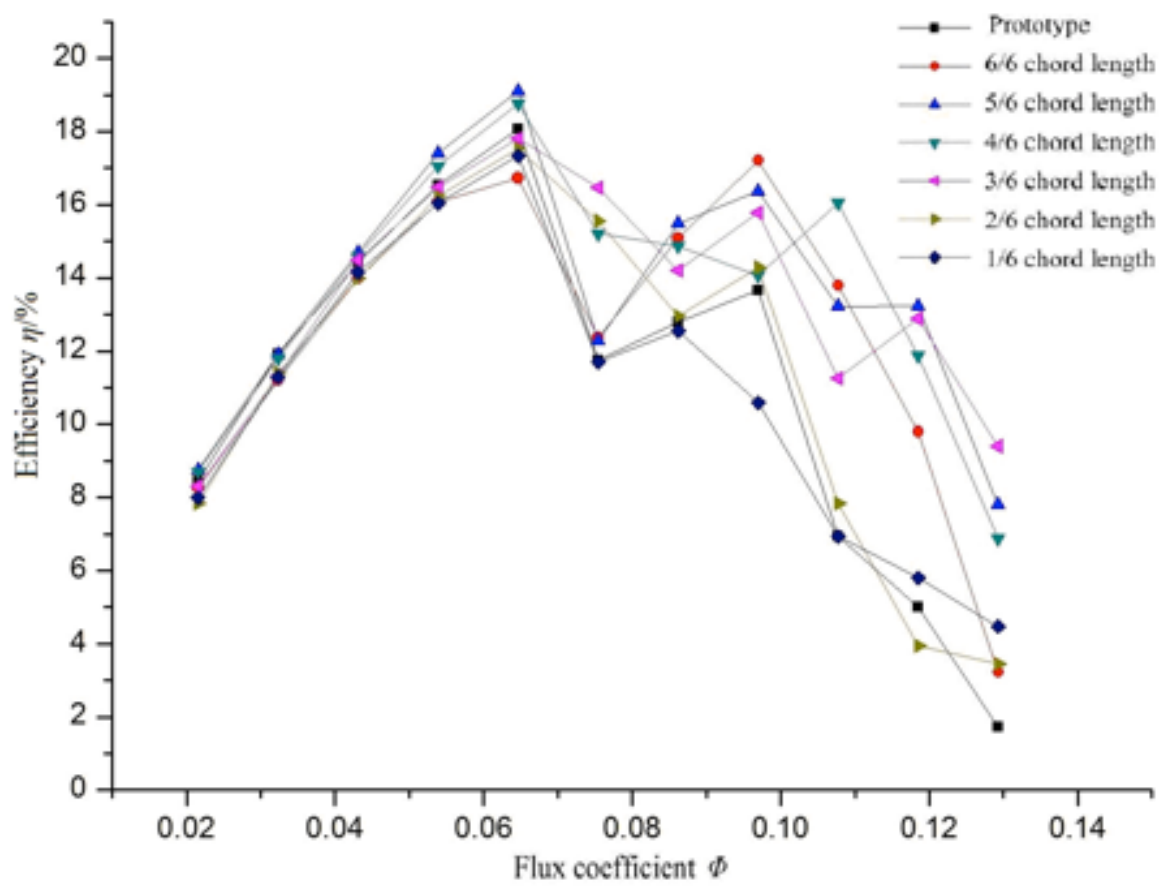

Fig. (6). Efficiency-Flux $(\eta-\Phi)$ dimensionless curves.

Compared efficiency values of the 7 fans, the fans with splitter blades are better than prototype fan, except that the efficiency of $1 / 6$ chord length is lower than that of prototype fan for high flux. Simultaneously, the efficiency of the 7 fans is similar for low flux.

Based on the above performance curve, it reveals that: (1) an appropriate chord length of splitter blades has a notable effect on enhancing the static characteristics of small axial fan, especially in high-flux operating point; (2) too long or too short chord length are detrimental to performance improvement, and the rough range from $2 / 6$ chord length to the $4 / 6$ chord length is optimum condition.

\subsection{Internal Flow Characteristics}

In order to understand the internal flow characteristics of fan, the static pressure contour and velocity contour are studied. Firstly, the meridional plane $(\mathrm{X}=0)$ of the computational domain is analyzed from the perspective of the pressure distribution. 
Fig. (7) is pressure contour (left) of meridional plane $(X=0)$ and the partial enlarged picture (right) of blade tip when it is the most effective point, namely, the flux coefficient $\Phi=0.0646\left(Q_{m}=0.006 \mathrm{~kg} / \mathrm{s}\right)$. From Fig. (7a) to (7f) (left), which are pressure contour of meridional plane $(X=0)$, it can be found:

(1) The pressure of inlet extension area is significantly higher than that of outlet extension area, and the pressure distribution in inlet extension area is relatively more uniform than that of outlet extension area;

(2) The static pressure increase of 6 model fans with splitter blades are higher than that of prototype fan, which is consistent with static characteristics mentioned by 4.2 in the same flux operating point.

From Fig. (7a) to (7f) (right), it can be found:

(1) The low pressure area occurs in blade root of suction side for the 7 fans;

(2) The pressure difference of pressure side and suction side at blade tip of 6 types of model fans are significantly smaller than prototype fan, which reduces emergence of backflow at blade tip clearance in a certain extent;

(3) The chord length of splitter blade is shorter, the pressure value of pressure side is higher. The reason is that shorter chord length weakens doing work ability of splitter blades, and pressure distribution gradually tends to that of prototype fan.

In order to further study the internal flow characteristics about fan, velocity distribution is analyzed next. Fluid flow is observed from four different axial cross-sections, which are cross-sectional $\mathrm{Z}=-6 \mathrm{~mm}$ (inlet of passage), $\mathrm{Z}=0$ (intermediate of passage), $Z=6 \mathrm{~mm}$ (outlet of passage), $Z=10 \mathrm{~mm}$ (exit of fan), respectively. Considering the length of paper, only takes the three optimum groups to demonstrate in this paper, that is, $2 / 6,3 / 6$ and $4 / 6$ chord length model.

(1) It is the prototype fan in Fig. (8). When fluid enters intermediate of passage, it appears a clear lowvelocity area near pressure side. With fluid flowing through passages of fan, low-velocity area becomes gradually smaller by doing work of blades. velocity area, which indicates that adding appropriate splitter blades can increase velocity of fluid in passage and suppress flow separation. Thus, the fluid flows more smoothly that will improve the performance of fan.

(3) Figs. (8b-11b) are the velocity distribution in intermediate of passage. There are high velocity regions near casing of prototype fan. However, the velocity of model fans is larger than that of prototype fan. Besides, the regions are located at the intermediate position between splitter blade and suction side of the adjacent long blade.

\subsection{Aerodynamic Noise Analysis}

Aerodynamic noise analysis will be studied from three respects that are the overall SPL, pressure fluctuation, power spectral density. Fig. (12) shows overall SPL of 6 model fans and prototype fan in 8 monitoring points. It can be seen from the figure:

(1) The overall SPL of the 6 model fans are lower than that of prototype fan at in 8 monitoring points, and it denotes that adding splitter blades has a relatively significant effect on reducing noise of fan.

(2) The overall SPL for the 6 model fans and prototype fan indicates that the chord length of splitter blades has a consistent trend of impact about noise. The overall SPL of monitoring point 2(ie, blade tip clearance) is maximal. In outlet extension area of computational domain, with increasing of the axial distance of monitoring points from fan, the overall SPL reduces gradually.

(3) Regarding monitoring point 1 and monitoring point 3 , their overall SPL are nearly the same.

(4) Except the overall SPL of 1/6 chord length, the noise reduction of model fans are relatively lower than that of prototype fan at each monitoring point. The other models significantly reduced and the most obvious is 4/6 chord length model.

Fig. (13) shows pressure fluctuation of 6 model fans and prototype fan in monitoring point 2 (i.e., blade tip clearance). It can be seen from the Fig. (13):

(1) The pressure fluctuation of 6 model fans is significantly lower than that of prototype fan on pressure fluctuation range. The pressure fluctuations range of prototype fan is $-70 \mathrm{~Pa} \sim 60 \mathrm{~Pa}$; at the peak, the peak values of 6 models are all lower than that of prototype fan by $40 \mathrm{~Pa}$; at the trough, the trough value of 6 models are all larger than that of prototype fan before 4000 steps, and the difference is not obvious after 4000 steps.

(2) The pressure fluctuation range of $3 / 6$ chord length model is smaller than that of other models, which indicates that splitter blades of this model is relatively better for improving the pressure fluctuation. Pressure fluctuation of $2 / 6$ chord length model and $4 / 6$ chord length model are smaller than that of other models except $3 / 6$ chord length model. The 1/6 chord length model is the most unapparent model about pressure fluctuation reducing.

Fig. (14a-f) shows power spectral density distribution of 6 model fans and prototype fan in monitoring point 2 (blade tip clearance). It can be seen from the figure:

(1) 6 model fans are significantly lower than the prototype fan on power spectral density.

(2) The peak value of power spectral density occurs at fundamental frequency and harmonics. With increasing of frequency, the peak value of power spectral density decreases gradually, and it disappears when 6 times and 7 times harmonics. 

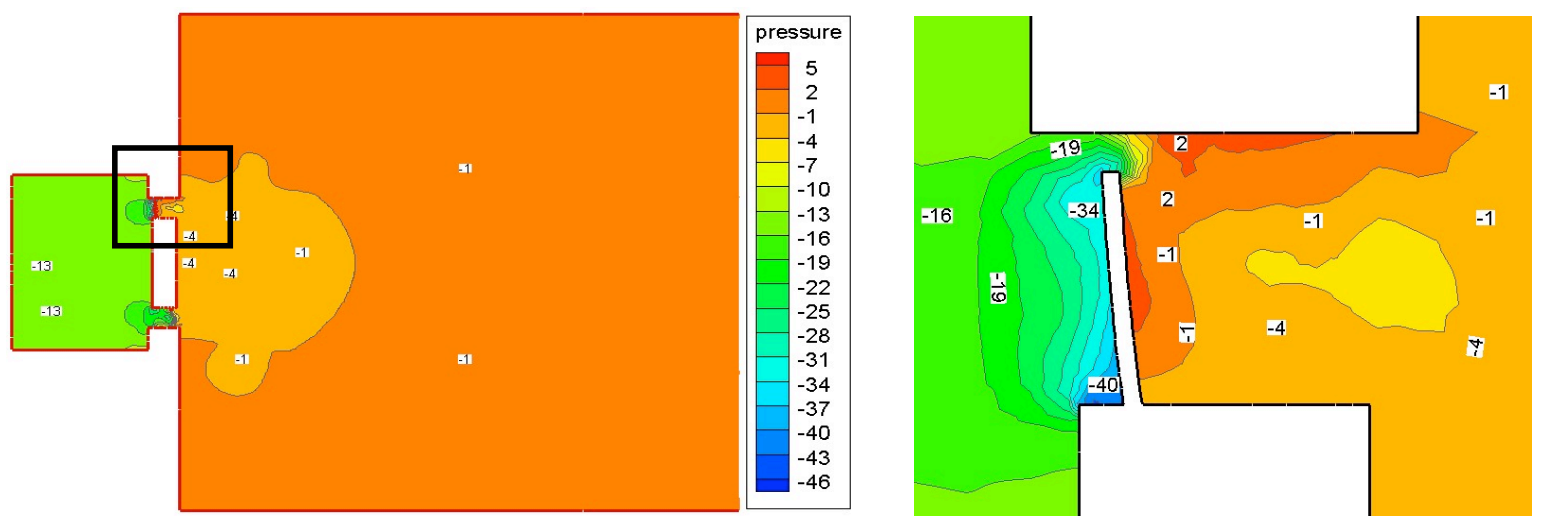

(a) prototype fan
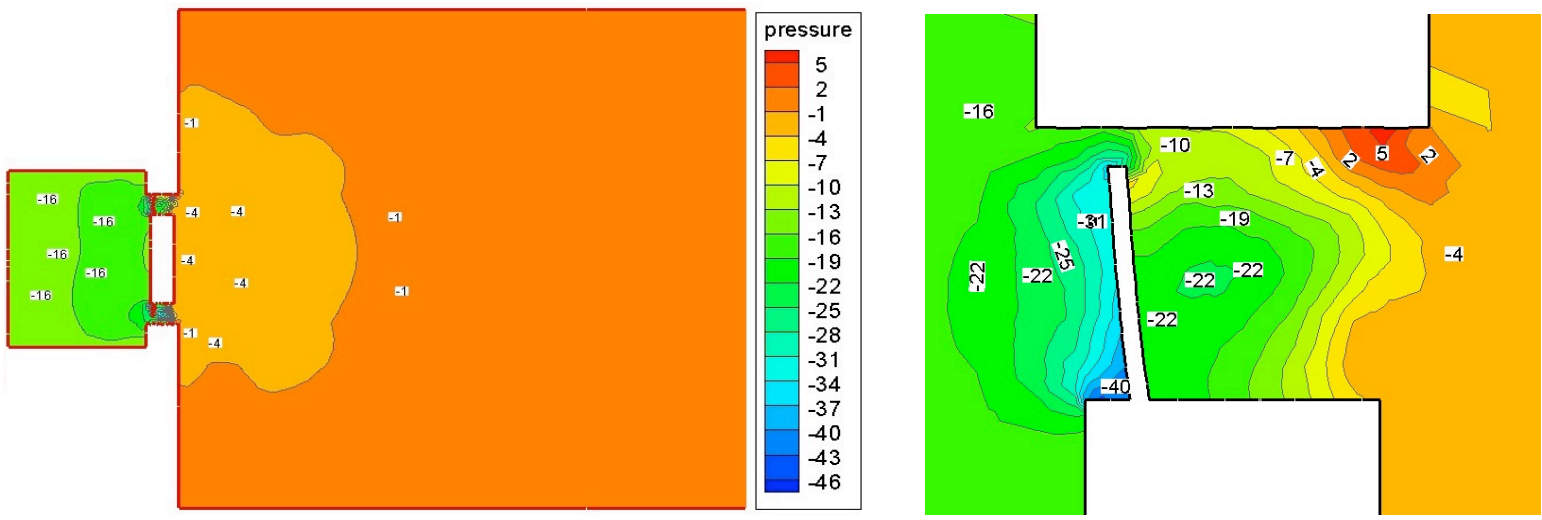

(b) $6 / 6$ chord length model
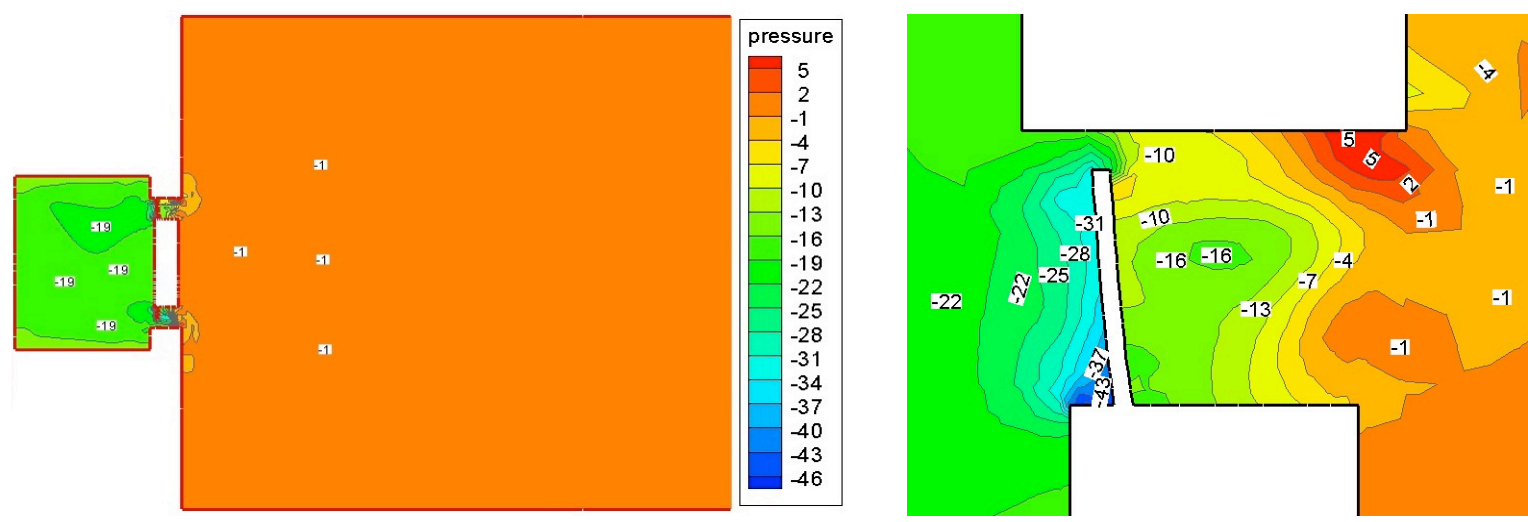

(c) $5 / 6$ chord length model
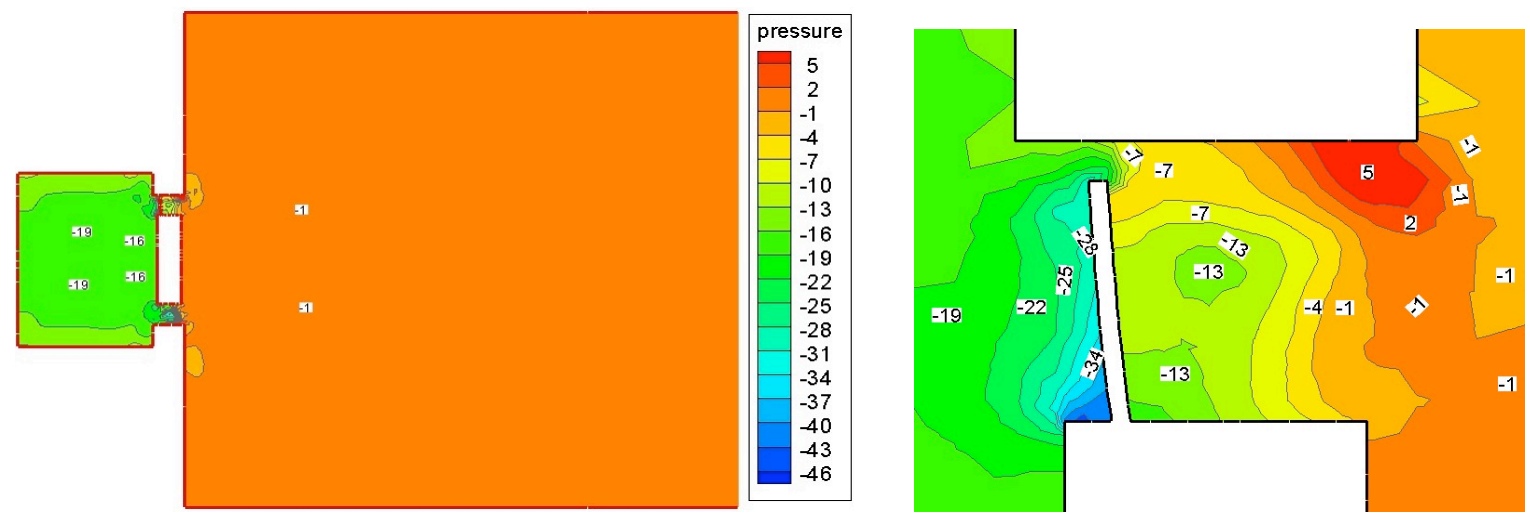

(d) $4 / 6$ chord length model 
(Fig. 7) contd.....
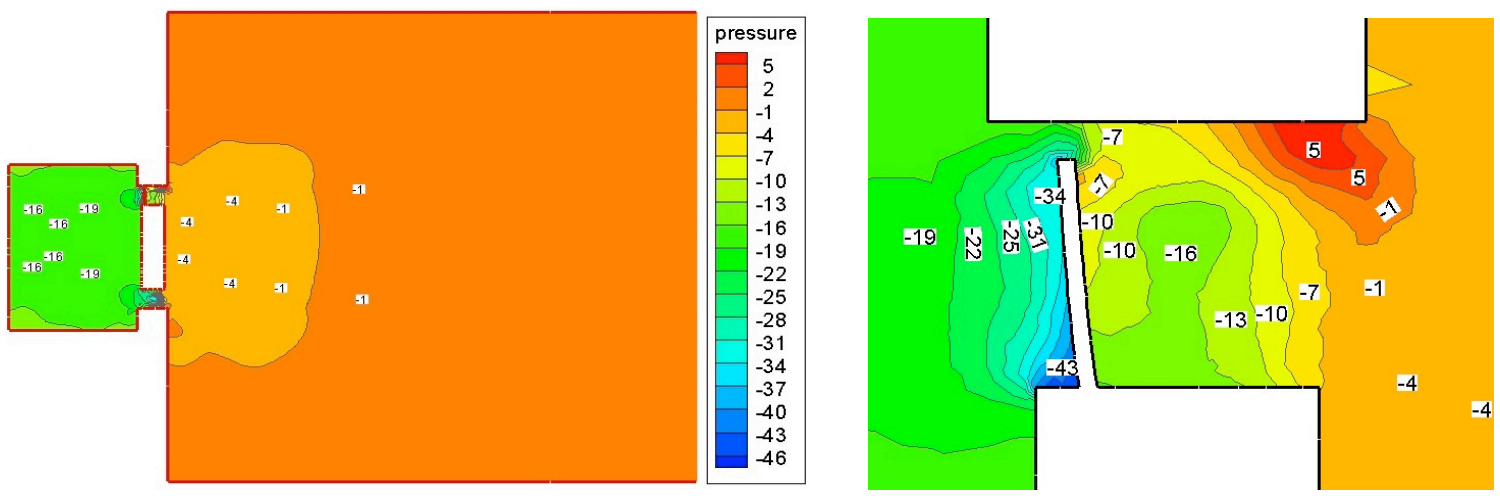

(e) 3/6 chord length model
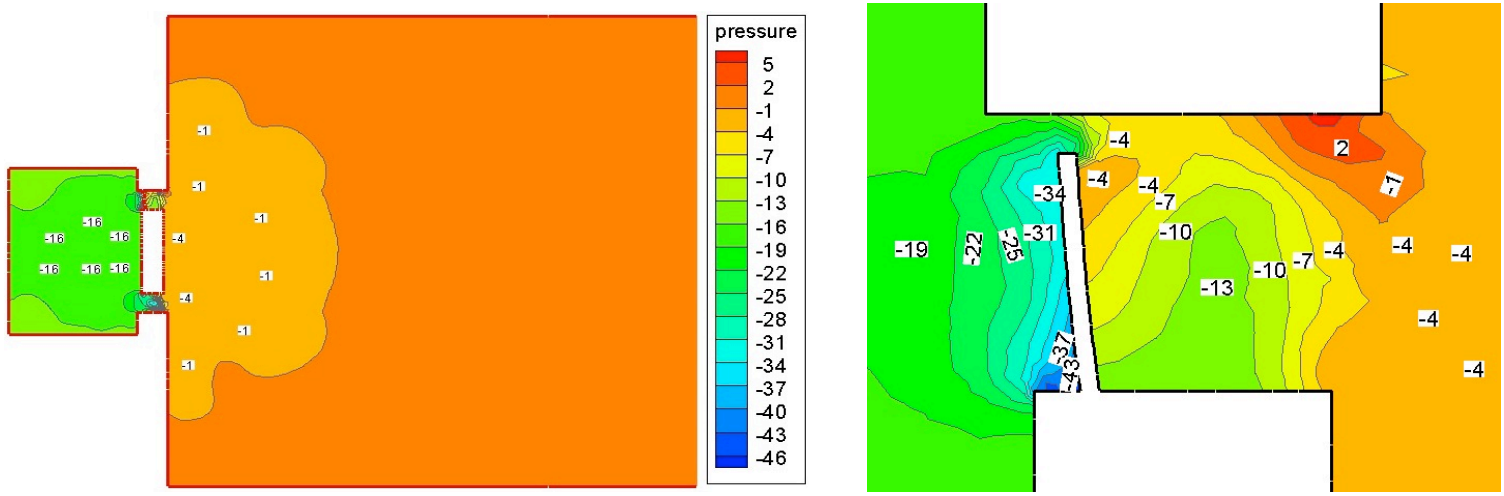

(f) $2 / 6$ chord length model
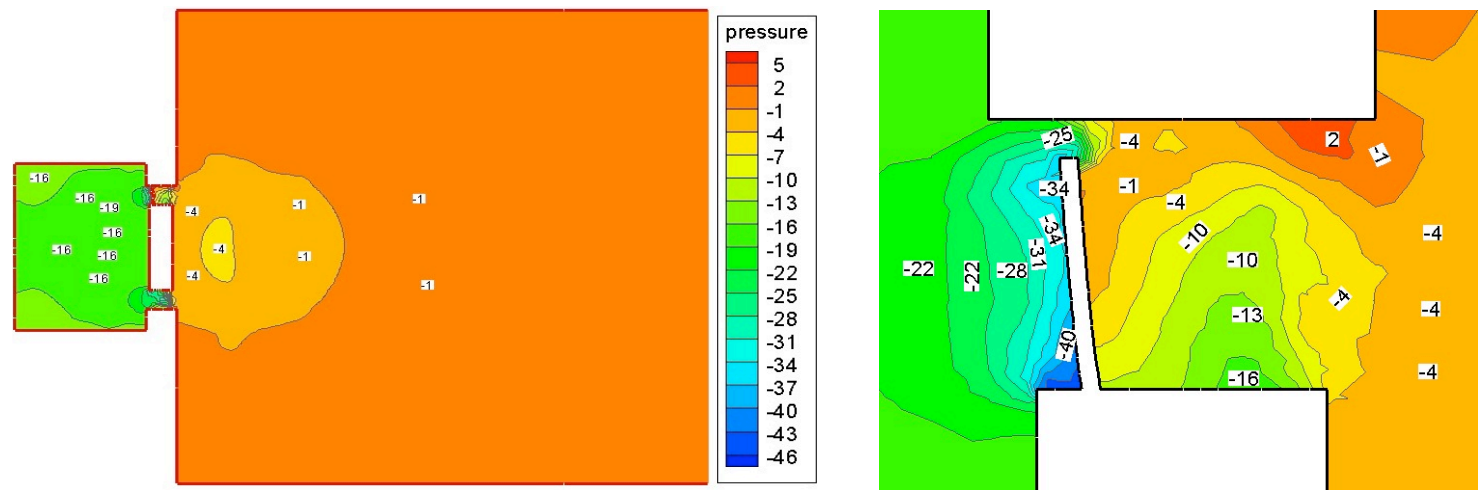

(g) 1/6 chord length model

Fig. (7). Pressure contour of meridional plane $(\mathrm{X}=0)$ and partial enlarged picture of blade tip.

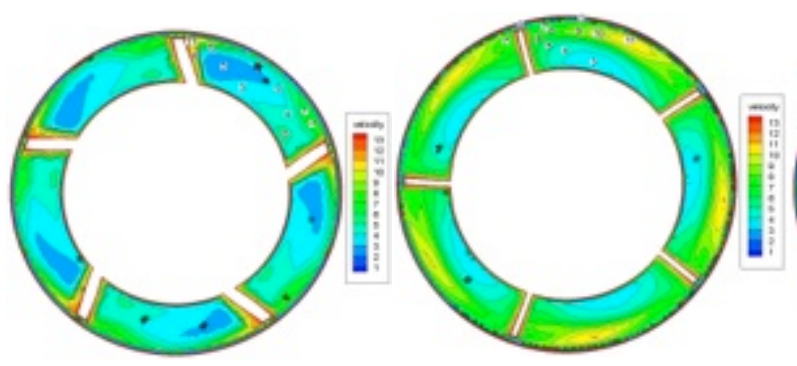

(a) Clip plane $Z=-6 \mathrm{~mm}$ (b) Clip plane $Z=0$

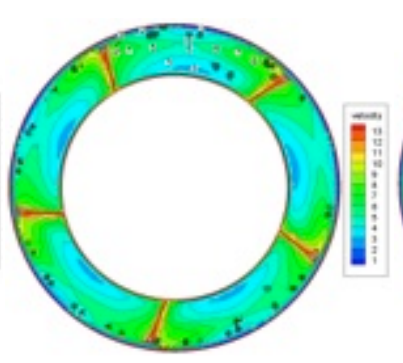

(c) Clip plane $Z=6 \mathrm{~mm}$

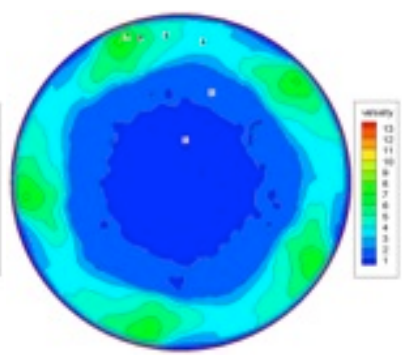

(d) Clip plane $Z=10 \mathrm{~mm}$

Fig. (8). Velocity contour from different axial cross-sections for prototype fan. 


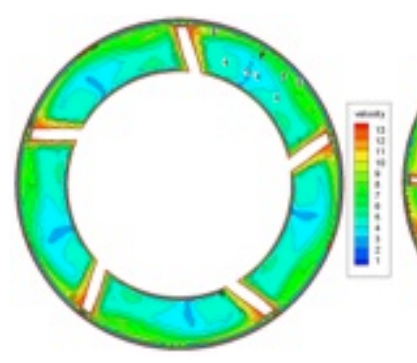

(a) Clip plane $Z=-6 \mathrm{~mm}$

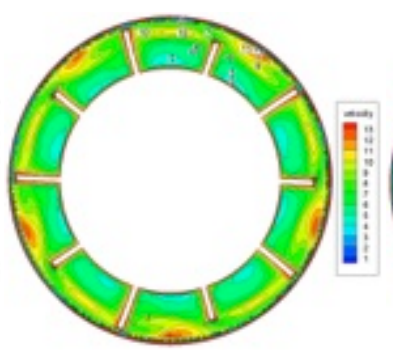

(b) Clip plane $Z=0$
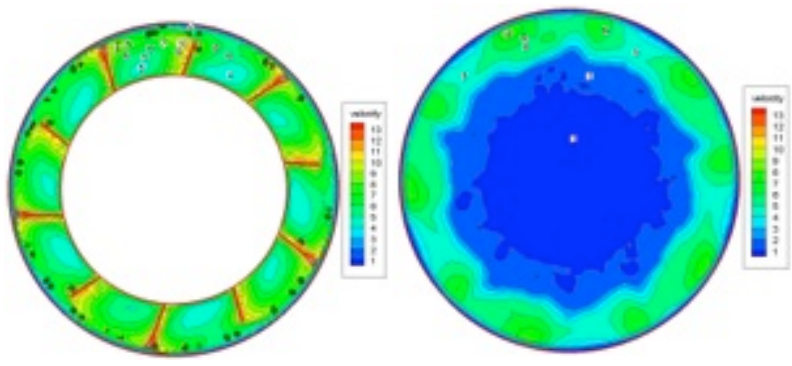

(c) Clip plane $Z=6 \mathrm{~mm}$

(d) Clip plane $Z=10 \mathrm{~mm}$

Fig. (9). Velocity contour from different axial cross-sections for $4 / 6$ chord length model.

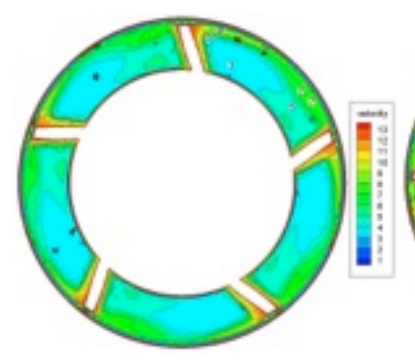

(a) Clip plane $Z=-6 \mathrm{~mm}$

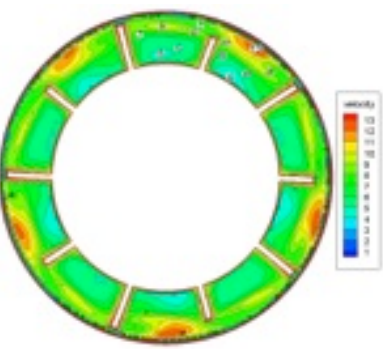

(b) Clip plane $Z=0$

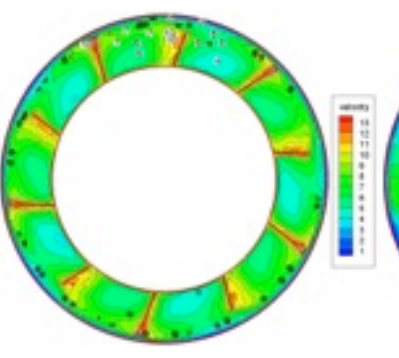

(c) Clip plane $Z=6 \mathrm{~mm}$

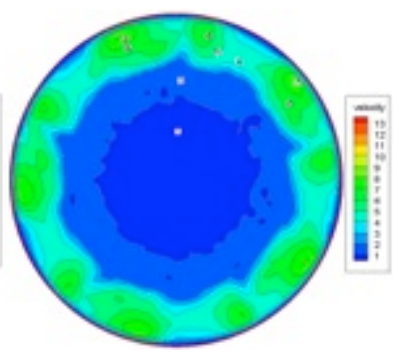

(d) Clip plane $Z=10 \mathrm{~mm}$

Fig. (10). Velocity contour from different axial cross-sections for $3 / 6$ chord length model.

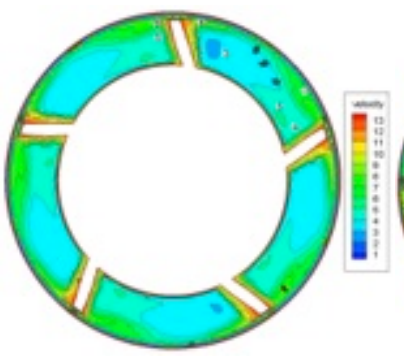

(a) Clip plane $Z=-6 \mathrm{~mm}$

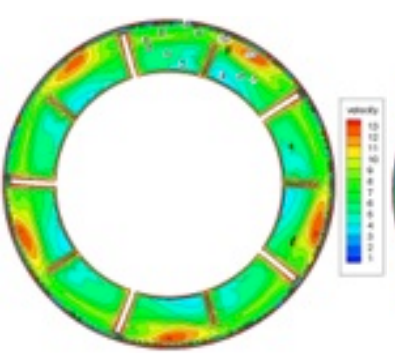

(b) Clip plane $Z=0$

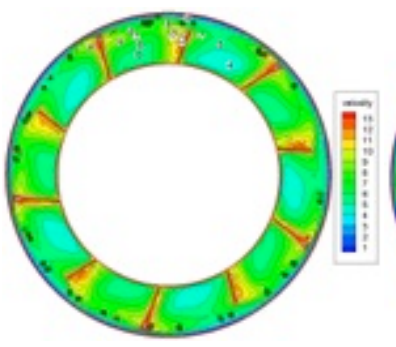

(c) Clip plane $Z=6 \mathrm{~mm}$

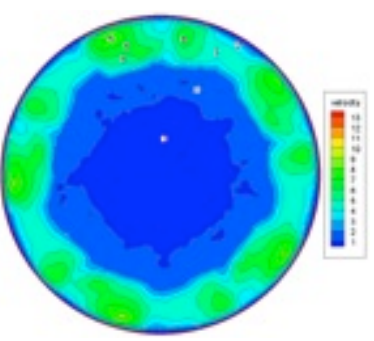

(d) Clip plane $Z=10 \mathrm{~mm}$

Fig. (11). Velocity contour from different axial cross-sections for $2 / 6$ chord length model.

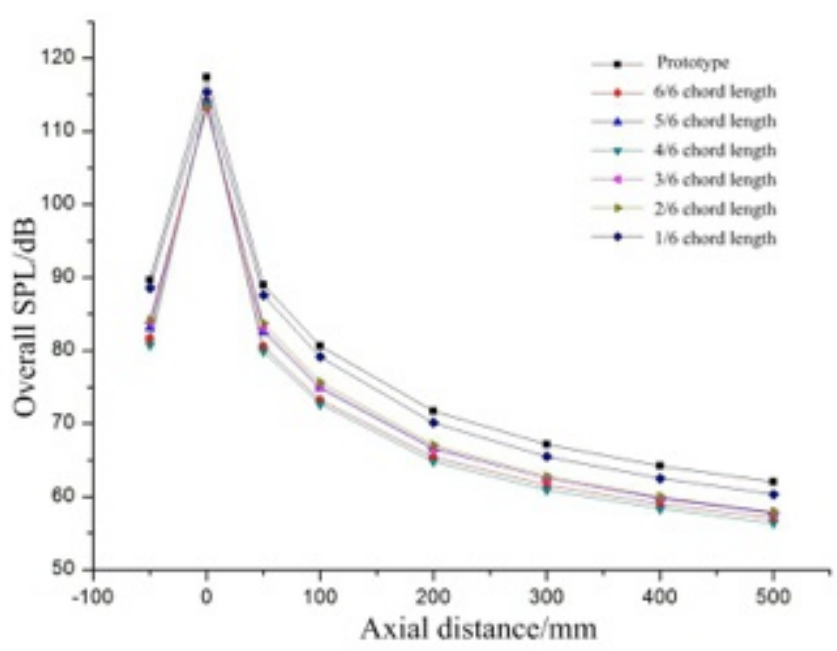

Fig. (12). Overall SPL for 8 monitoring points.

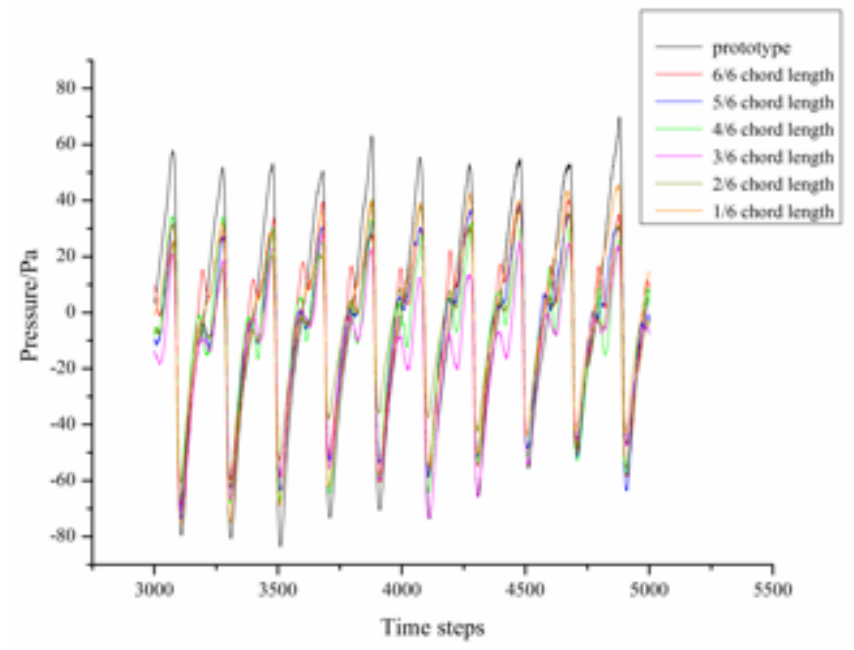

Fig. (13). Pressure fluctuation of blade tip clearance. 


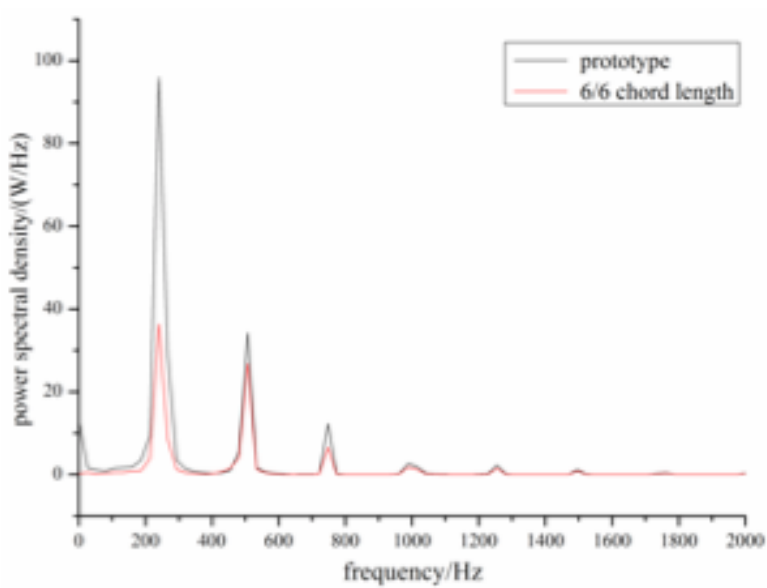

(a) 6/6 chord length

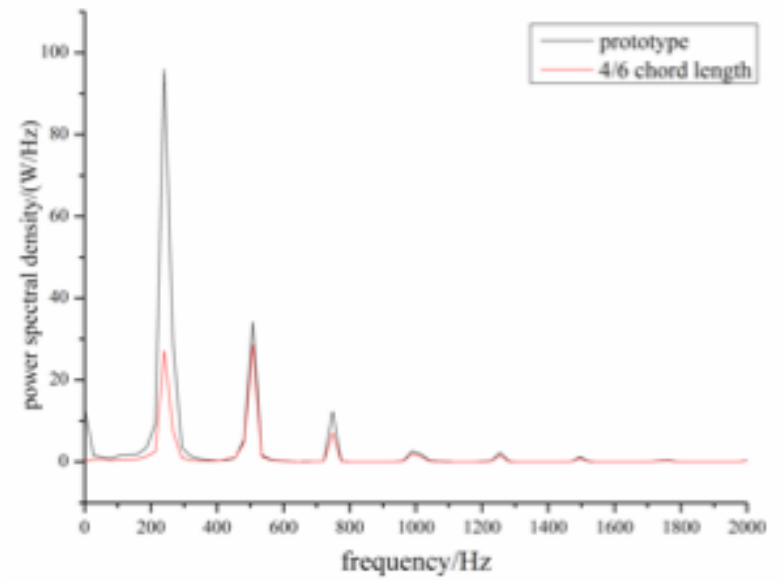

(c) $4 / 6$ chord length

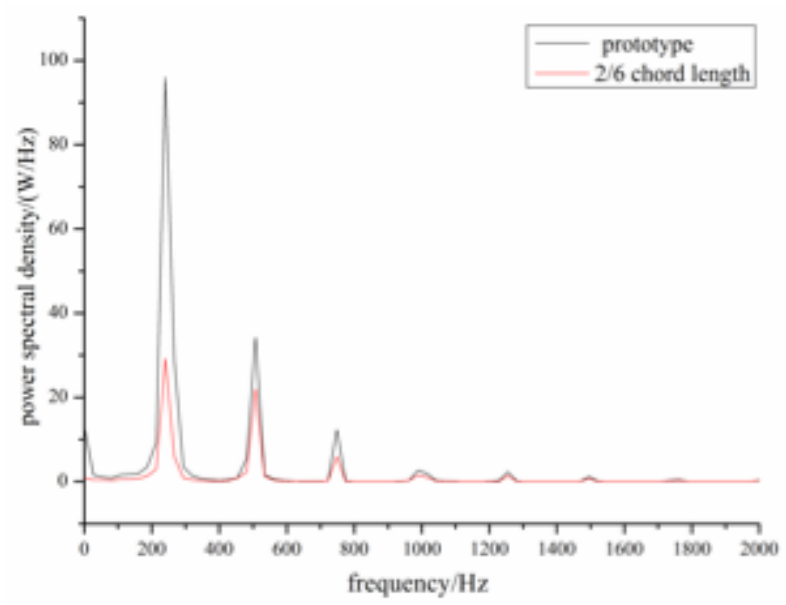

(e) $2 / 6$ chord length

Fig. (14). Power spectral density distribution (monitoring point 2).

(3) At the fundamental frequency, 3/6 chord length model reduces most obviously on peak of power spectral density, and followed by $4 / 6$ and $2 / 6$ chord length model. Besides, the smallest decrease is $1 / 6$ chord length model on peak of power spectral density, it denotes that adding appropriate splitter blades is effective to decreasing the power spectral density.

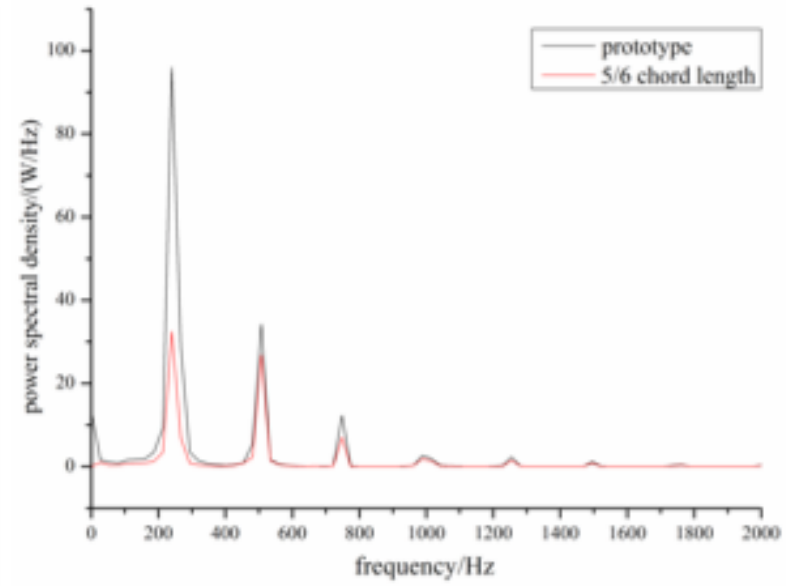

(b) $5 / 6$ chord length

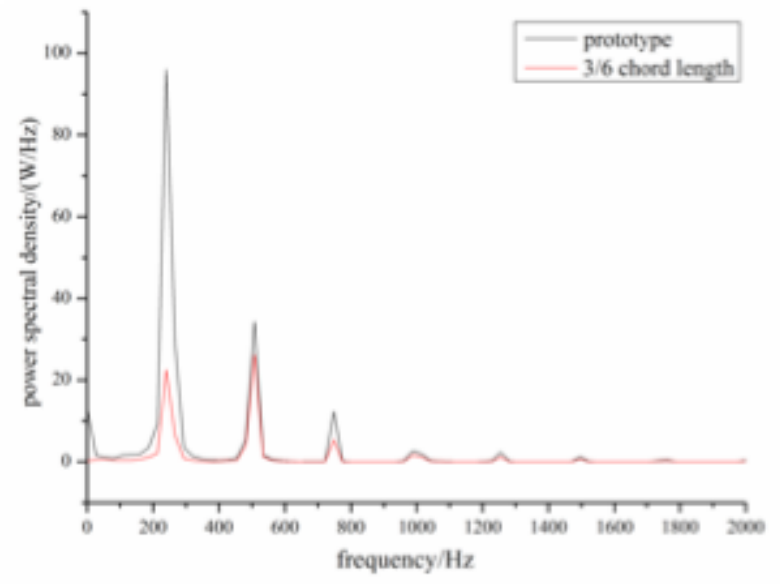

(d) $3 / 6$ chord length

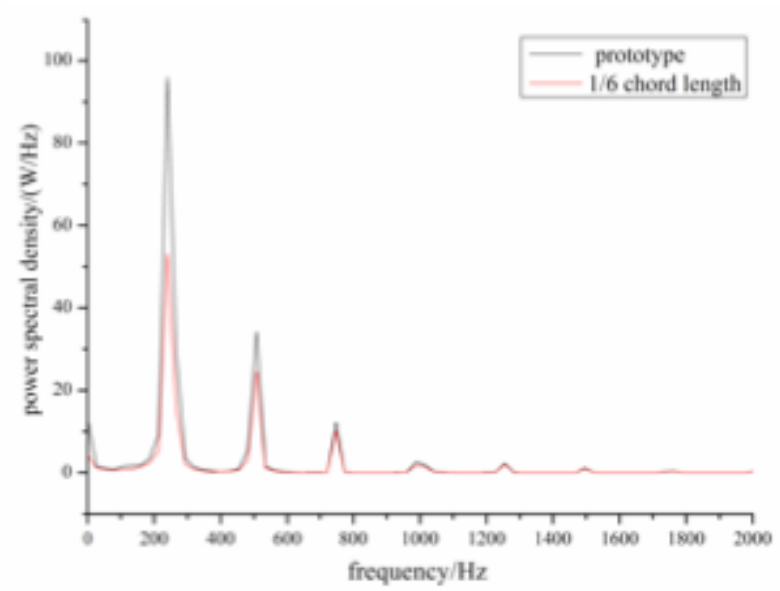

(f) $1 / 6$ chord length

Above analysis shows: (1) fans with different chord lengths splitter blades are better than prototype fan about aerodynamic noise characteristics; (2) splitter blade with suitable chord length has a significant effect on improving aerodynamic noise characteristics; (3) comprehensive comparison about fans with splitter blades, it reveals that $2 / 6,3 / 6$ and $4 / 6$ chord length model have relatively better acoustic characteristics. 


\section{CONCLUSION}

This paper has established 6 types of small axial fan models with different chord lengths splitter blades and compared with the prototype fan. Besides, their static characteristics, internal flow and aerodynamic acoustic characteristics are explored, and the effect of chord length for splitter blades on performance of fan is analyzed. And it draws the following conclusions:

(1) The static characteristics of 6 models with different chord lengths splitter blades are better than that of prototype fan. Splitter blades with appropriate chord length have a certain effect on enhancing static characteristics. Specifically, static characteristics of $2 / 6,3 / 6$ and 4/6 chord length model are better than that of other models.

(2) After adding splitter blades of different chord lengths, the internal flow is significantly improved. Besides, it makes pressure distribution of blade surface more uniform, and it is conducive to inhibit backflow that from pressure side to suction side at tip and the leading edge of the longer blade.

(3) The fans with different chord lengths splitter blades are better than prototype fan about aerodynamic noise characteristics. Splitter blade with suitable chord length has a significant effect on improving aerodynamic noise characteristics. The result reveals that $2 / 6$, $3 / 6$ and $4 / 6$ chord length model have relatively better acoustic characteristics.

\section{CONFLICT OF INTEREST}

The authors confirm that this article content has no conflict of interest.

\section{ACKNOWLEDGEMENTS}

This work is supported by Natural Science Foundation of China (No. 51276172) and National Science and Technology Support Project (No. 2013BAF05B01). Simultaneously, Zhejiang Science and Technology Plans Public Projects (No. 2014C31116), the Special Major Project of Science and Technology of Zhejiang Province (No. 2013C01139), and the Fluid Engineering Innovation Team of Zhejiang SciTech University (ZSTU) (No. 11132932611309) are also offering support to this research.

\section{REFERENCES}

[1] S. C. Lin, M. L. Tsai, "An integrated performance analysis for a small axial-flow fan", Proceedings of the Institution of Mechanical Engineers, Part C: Journal of Mechanical Engineering Science, vol. 224, pp. 1981-1994, 2010.

[2] S. C. Lin, M. L. Tsai, "An integrated study of the design method for small axial-flow fans, based on the airfoil theory", Proceedings of the Institution of Mechanical Engineers, Part C: Journal of Mechanical Engineering Science, vol. 225, pp. 885-895, 2011.

[3] B. Li, H. Qian, L. Zhang, Y. Jin, "Aerodynamic performance study on small axial fan with perforation blade", Journal of Zhejiang SciTech University, vol. 30, pp. 76-80, 2013.

[4] S. Kang, R. Guo, B. Tang, "Numerical simulation of a smallscale axial cpu fan", Journal of Engineering Thermophysics, vol. 30, pp. 1853-1855, 2009

[5] P. Yuan, J. Hu, Z. Wang, "Design and flow investigation of a centrifugal impeller with splitter blade", Gas Turbine Experiment and Research, vol. 21, pp. 33-37, 2008.

[6] S. Luo, Z. Cai, "Changing blade number and long-short blade structure to the effect of 3-D viscous flow field in centrifugal impelle", Compressor Blower \& Fan Technology, pp. 3-6, 2000.

[7] L. Gui, C. Gu, H. Chang, "Influences of splitter blades on the centrifugal fan performances", ASME, The Gas Turbine Aero engine Congress and Exposition, 1989, pp. 1-6. 